\title{
Side Lobe Reduction in an X - Band Horn Antenna Loaded by a Wire Medium
}

\author{
Antônio Tomaz¹, Joaquim J. Barroso², Ugur Cem Hasar³
}

\begin{abstract}
Through electromagnetic simulation, the present work reports on a comparative study of the enhanced radiation properties of a standard X-band horn antenna loaded by a wire medium. Acting as an artificial dielectric, the wire medium consists of an array of parallel metallic wires installed into the antenna with the wires oriented in the direction of the incident electric field. As compared with the properties of the air horn antenna, the wire-medium antenna produces an appreciable reduction in the side-lobe levels with an improvement of $10 \mathrm{~dB}$ at $8.87 \mathrm{GHz}$ in the first side lobe. For the wire-medium antenna, the $E$ - and $H$-plane radiation patterns look similar, a property desired for precision radar and radiometric mapping systems.
\end{abstract}

KEYWORDS: Wire medium, Artificial dielectric, $X$ - band horn antenna, Sidelobe reduction, Directivity.

\section{INTRODUCTION}

A dense array of identical conducting wires arranged in a periodic pattern embedded in a lightweight dielectric matrix constitutes an artificial electromagnetic structure which is generally termed as wire medium metamaterial (Simovski et al., 2012), in that such a structure can be engineered to exhibit properties not found in naturally occurring materials. Recent studies have shown that wire medium arrays can provide an interesting approach to improve the radiation performance of microwave antennas (Eleftheriades and Engheta 2011; Lier et al. 2011). A properly designed wire medium can behave as a homogenized artificial dielectric having a relative permittivity less than unity. As the index of refraction $n$ relates to the relative permittivity $\varepsilon_{r}$ via $n=\sqrt{\varepsilon_{r}}$, such artificial medium exhibits near-zero refractive index, which enables the realization of highly-directive beams (Xiao and Xu 2009; Wu et al. 2007; Tomaz and Barroso 2013; Enoch et al. 2002; Sahu et al. 2014). While previous studies have focused on gain and directivity enhancement of wire-medium antennas (Hrabar et al. 2009; Franson and Ziolkowski 2009; Lovat et al. 2006; Tomaz et al. 2013), here we present a conceptual study of a wire metamaterial designed to reduce the level of side lobes while still enhancing the radiation characteristics of a standard X-band horn antenna. Antennas with improved side lobe performance are desirable for many directive applications, such as in radar, direction-finding equipment, and in systems to reject interference sources (Semplak 1983).

The rest of the paper is organized as follows. In "Wire medium structure" section, we discuss the conditions under which a wire medium, i.e. a set of identical metallic wires arranged in

1.Instituto Tecnológico de Aeronáutica - Divisão de Engenharia Eletrônica - Departamento de Microondas e Optoeletrônica - São José dos Campos/SP - Brazil. 2. Instituto Nacional de Pesquisas Espaciais - Laboratório Associado de Plasma - São José dos Campos/SP - Brazil. 3.University of Gaziantep - Department of Electrical and Electronics Engineering - Gaziantep - Turkey.

Author for correspondence: Joaquim J. Barroso | Instituto Nacional de Pesquisas Espaciais | Avenida dos Astronautas, 1.758 - Jardim da Granja | CEP: $12.227-010$ São José dos Campos/SP - Brazil | Email: barroso@plasma.inpe.br

Received: 02/04/2015 | Accepted: 08/11/2015 
a periodic pattern, can be modeled as a homogeneous anisotropic material described by a local effective permittivity. On the basis of such a description, an artificial dielectric slab is then designed in "Wire medium simulation" section to exhibit an effective refractive index of 0.6 at $8.87 \mathrm{GHz}$. The effects of inserting the designed wire medium into an X-band antenna are reported in "Electromagnetic simulation of the wire-medium loaded antenna" section. The main effect is a reduction of side lobes, which is explained as a result of refractive effects arising from the less-than-unity index of refraction. The rays emitted from the antenna are bent toward broadside in the air region, where an angular confinement of the output radiation is observed. "Conclusions" section summarizes our work and describes quantitative features of the side lobe reduction.

\section{WIRE MEDIUM STRUCTURE}

A metamaterial wire medium can be designed by analytical models supported by full-wave electromagnetic simulations to meet specific requirements. In our study, the wire medium consists of thin metallic wires of diameter $d$ and is arranged on a square lattice of a periodic spacing a as shown in Fig. 1.

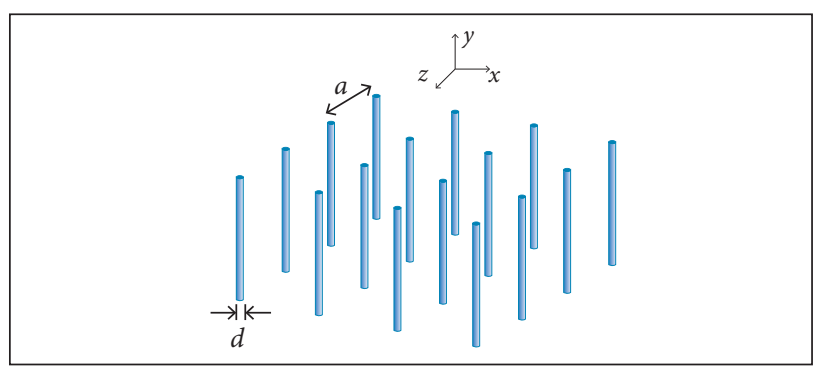

Figure 1. Wire medium structure.

Provided that the wire radius and the periodic distance are very small in comparison with the wavelength inside the structure and for the case of plane-wave incidence with the electric field parallel to the wires, the wire medium can be described as a homogeneous artificial dielectric with relative permittivity given by Simovski et al. 2012:

$$
\varepsilon_{r}=\varepsilon_{h}\left(1-\frac{f_{c}^{2}}{\varepsilon_{h} f^{2}}\right)
$$

where:

$\varepsilon_{h}$ is the permittivity of the host medium, $f$ is the frequency of the incident wave, and $f_{c}$ is the equivalent cutoff frequency, which is determined by calculating the local field that excites each wire and then summing up all the fields generated by the wires in the infinite periodic array (Belov et al. 2002; Maslovski et al. 2002).

$$
f_{c}=c\left[a \sqrt{2 \pi\left(\ln \frac{a}{\pi d}+0.5275\right)}\right]^{-1}
$$

where:

$c$ is the speed of light.

The wire medium is envisaged to operate in the $\mathrm{X}$ - band $(8.2-12.4 \mathrm{GHz})$ and, for achieving optimum frequency response, the geometrical parameters should be properly chosen. The lattice constant was set then to $a=10.0 \mathrm{~mm}$ and, for the wire diameter, a commercially available value of $d=0.5 \mathrm{~mm}$ was chosen. Using Eq. 2, these parameters give the cutoff frequency of $7.7 \mathrm{GHz}$. For other values of $d$ and $a$, the corresponding cutoff frequencies are given by isofrequency curves shown in Fig. 2.

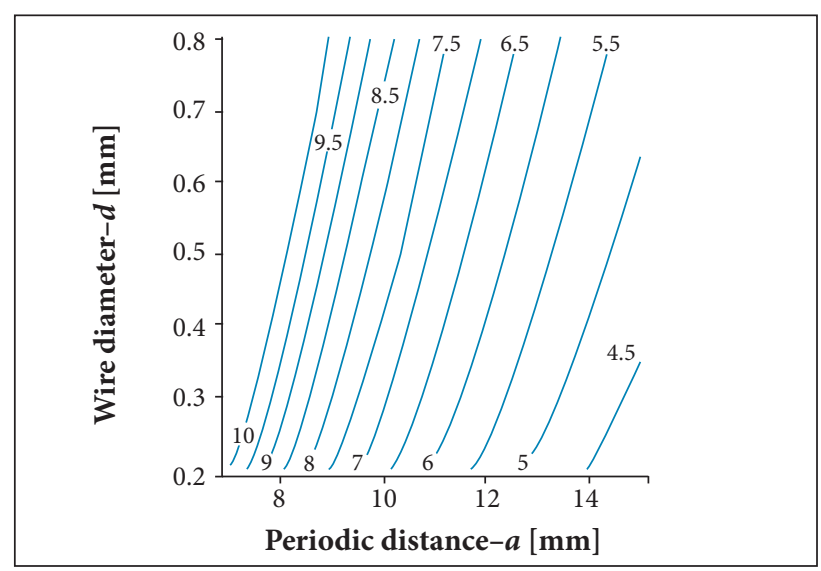

Figure 2. Curves of constant cutoff frequencies $[\mathrm{GHz}]$ on the plane of parameters a-d.

We see that $f_{c}$ is very sensitive to the lattice constant; at $d=0.5 \mathrm{~mm}$, for instance, $f_{\mathrm{c}}$ increases from 7.7 to $10.0 \mathrm{GHz}$ when $a$ decreases from 10.0 to $8.1 \mathrm{~mm}$. Therefore, for a constant $d$, an increase in lattice constant $a$ is accompanied with a decrease $\operatorname{in} f_{c}$. This circumstance is very similar to that of linear array of antennas (Balanis 2005).

In concluding this section, it is worth noting that Eq. 1 applies for the case when the electric field of the incident plane wave is parallel to the wires (ordinary mode). For the cases where the electric field is no longer parallel to the wires, the medium is known to exhibit spatial dispersion (Belov et al. 2003). In this situation the wave vector has a component parallel to the wires 
(referred to as longitudinal or extraordinary mode), giving rise to periodic accumulation of charge and so the divergence of the electric field is no longer zero. Longitudinal modes are highly dispersive implying strong non-locality of the system for which Eq. 1 does not hold. In the electromagnetic simulations to be presented in the next section, the incident plane wave propagates perpendicular to the wires; in this case Eq. 1 does apply and is then used to predict the frequency dispersive behavior of the wire medium.

\section{WIRE MEDIUM SIMULATION}

The analytical model was verified through electromagnetic full-wave simulations by using the CST MWS software (CST Microwave Studio 2014) to ascertain the behavior of the artificial dielectric formed by a periodic array of metallic wires. As displayed in Fig. 3a, the simulated structure is composed of five $0.5 \mathrm{~mm}$ diameter wires evenly spaced at $10.0 \mathrm{~mm}$.

In the simulations, electric and magnetic boundary conditions were applied on the walls perpendicular and parallel to the wires, respectively. Two open ports were used to simulate the scattering $S$-parameter response to a normally incident plane wave with the electric field polarized along the

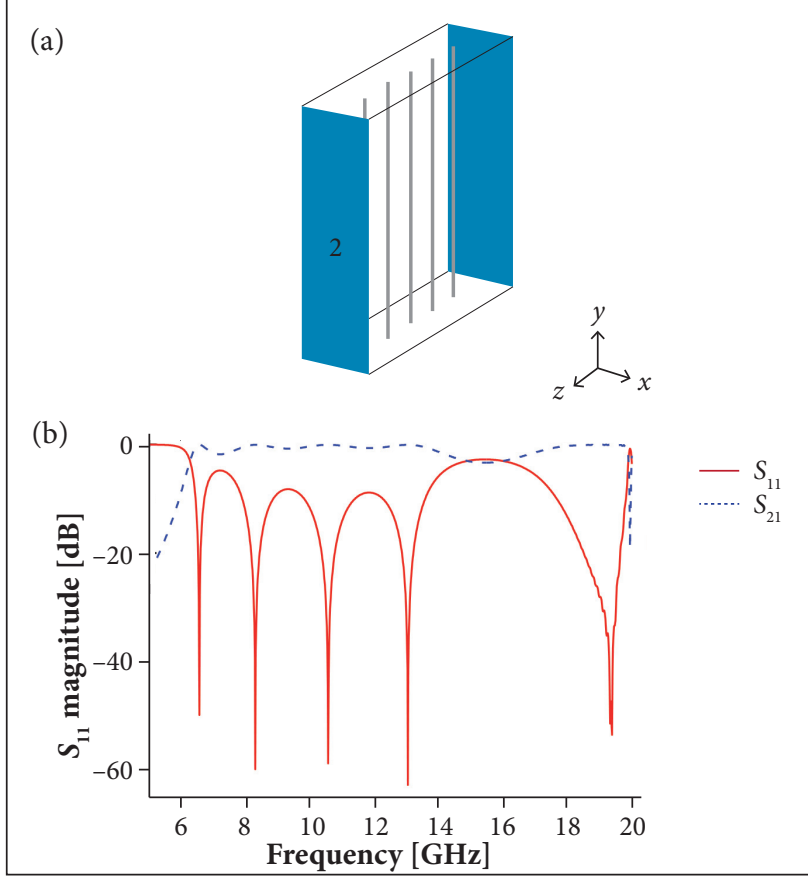

Figure 3. (a) Simulation setup of the periodic array of five wires; (b) Magnitude of scattering parameters $S_{11}$ and $S_{21}$. wires. Figure $3 \mathrm{~b}$ shows the simulated scattering parameters $S_{11}$ and $S_{21}$ of the periodic structure. In addition to a transmission band starting at about $7.0 \mathrm{GHz}$ and extending up to $14.0 \mathrm{GHz}$, four resonance dips are noticed. The occurrence of four dips arises from the fact that five periodic layers constitute an array of four coupled resonators (Barroso et al. 2014). Beyond the pass band gap, the region starting from $13.0 \mathrm{GHz}$ and extending up to $19.0 \mathrm{GHz}$ is the Bragg band gap. Increasing the number of wires, e.g. to 20, would make the gap transition much sharper and just starting at $15.0 \mathrm{GHz}$, which is the Bragg frequency $-f_{B}(\mathrm{GHz})=15 / a(\mathrm{~cm})-$ for the design period $a=1.0 \mathrm{~cm}$.

From simulated scattering parameters and using the retrieval procedure described in Hasar and Barroso (2011), the retrieved refractive index is shown in Fig. 4. Then we see that the effective index of refraction for the wire medium so designed has an average value of 0.62 over the X-band. Point $P$ at $7.76 \mathrm{GHz}$ corresponds to the plasma cutoff frequency, which explicitly appears in Eq. 1 of the dispersive model. Point $B$ at $15.0 \mathrm{GHz}$ indicates the Bragg frequency, at which the refractive index is close to 1.0. The dispersive model fails above this frequency, so that diffraction effects dominate and the retrieved parameters produce unphysical artifacts as evidenced by the negative slope between 15.0 and $17.7 \mathrm{GHz}$. It is interesting to notice that, for frequencies below $7.76 \mathrm{GHz}$ (to the left of point $P$ ), the wire medium can be regarded as a polarizer, in the sense that the verticallyoriented incident field is reflected backwards. This is because the impinging electric field, which is aligned parallel to the wires, makes the free electrons to surge up down in the wire grid which then behaves like a metal reflector. For an electric field perpendicular to the wires, the electrons cannot move

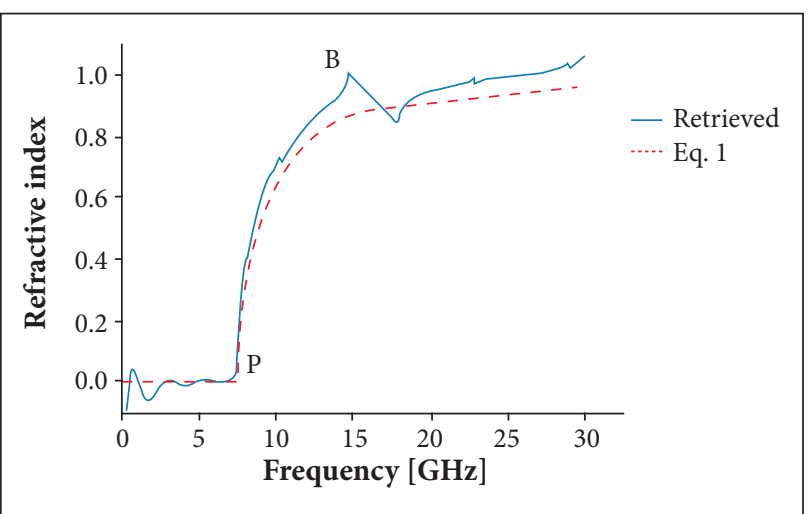

Figure 4. Retrieved real part of the refractive index and analytical curves from the Drude model calculated by using Eq. 1. 
as easily across the thin wires and the incident field passes freely through the grid.

The angular bending of rays emitted from a source immersed in a medium with a refractive index $(n)$ with a near-zero value is illustrated in Fig. 5. Because $n \approx 0$, all the rays emitted from the source would refract, by Snell's law, almost perpendicular to the material surface. It is noticed in Fig. 4 that to the frequency around $9.0 \mathrm{GHz}$ there corresponds a refractive index of 0.6. In the next section we verify how such refractive effects show up in a standard rectangular horn antenna.

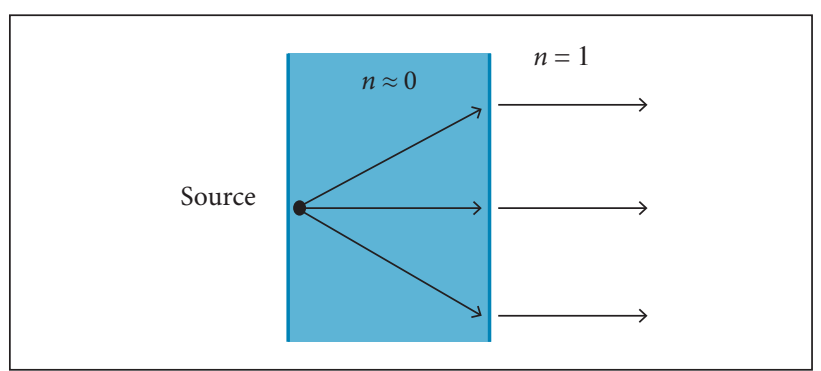

Figure 5. Refraction on the interface of a near-zero refractive index medium.

\section{ELECTROMAGNETIC SIMULATION OF THE WIRE-MEDIUM LOADED ANTENNA}

A simulation setup (Fig. 6) was realistically implemented in the CST Microwave Studio (CST Microwave Studio 2014; Datasheet Advanced Technical Materials, Inc. 2012). The structure was modelled as shown in Fig. 6, using $6.5 \times 10^{6}$ meshcells with a grid resolution of 30 steps per wavelength, which ensured the convergence properties of the fields. Five rows of the wire medium previously discussed are loaded in the horn antenna with geometrical details shown in Fig. 6. The arrays of metallic wires are accommodated and fixed in a Styrofoam plate $10.0 \mathrm{~mm}$ thick. The reason for using this material to host the metallic wires within the antenna is that it has electromagnetic properties similar to those of air medium (a relative permittivity of 1.03 and loss tangent of $1.0 \times 10^{-4}$ ) (Barker-Jarvis et al. 2001) so that it directly allows us to compare the radiation characteristics of the wire-loaded horn antenna with those of the air horn antenna.

The magnitudes of the scattering parameter $S_{11}$ of the wire medium along with the air horn are demonstrated in Fig. 7. We see that four resonance dips are lower than $-12 \mathrm{~dB}$ over the

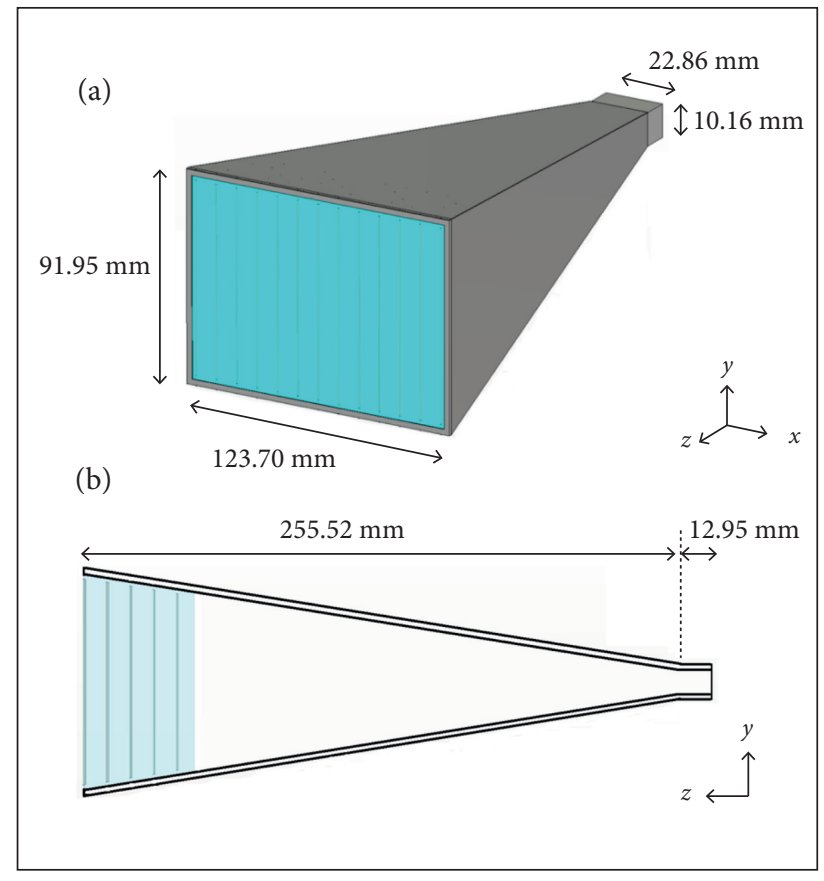

Figure 6. Horn antenna (Datasheet Advanced Technical Materials, Inc. 2012) loaded with the wire medium. (a) Perspective view; (b) Cut-away view.

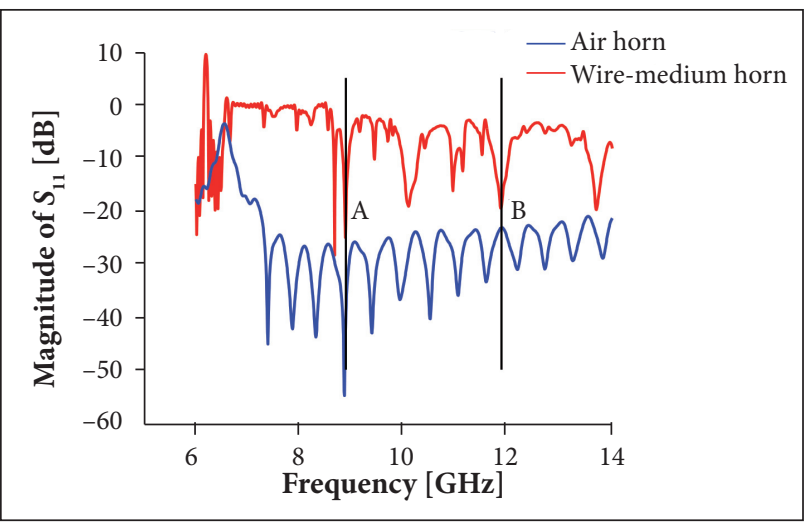

Figure 7. Simulated $S_{11}$ parameter magnitude for the air horn and wire-medium antennas. Dips $A$ and $B$ marked by the vertical lines correspond to 8.87 and $11.86 \mathrm{GHz}$.

$8.2-12.4 \mathrm{GHz}$ range (X - band), which implies that impedance matching bandwidth of the antenna is achieved only at these dips. This behavior in the $\left|S_{11}\right|$ curve differs greatly from that of the air horn, for which the $\left|S_{11}\right|$ level stays below $-25 \mathrm{~dB}$ in the whole frequency range.

Associated with the five loaded layers, five resonance dips are identified at $8.65,8.87 ; 10.08 ; 10.93$ and $11.86 \mathrm{GHz}$ below $-15 \mathrm{~dB}$. Not present in the five-layer structure in Fig. 3, which shows four resonant dips, there appears an extra mode in Fig. 7. 
The lowest-frequency $8.65 \mathrm{GHz}$ mode in Fig. 7 is sustained by back and forth round-trip reflections arising from the interfaces at the horn-waveguide junction and at the innermost wire layer. Nevertheless, apart from the extra dip associated with the trapped mode, the $S_{11}$ plots in Figs. 3 and 7 agree with respect to the number of resonance dips, albeit they markedly differ both in depth and shape. This difference is primarily due to the influence of the slanted metal plates in the pyramidal horn antenna. Moreover, while in the modeled antenna the wires do not touch the flared horn, in the parallel-plate waveguide (Fig. 3), the wires are connected to the top and bottom plates, and by image theory this system is equivalent to an array of infinitely long wires.

The radiation patterns at $8.87 \mathrm{GHz}$ for the air horn and wiremedium antennas are compared in Fig. 8. It is clearly apparent in Fig. 8a that the E-plane radiation pattern for the wire-loaded antenna yields significantly reduced side lobes, with the first side lobe down $24 \mathrm{~dB}$ from the main lobe. In addition, Fig. $8 \mathrm{~b}$ shows an appreciable reduction in the backside radiation in the $H$-plane. Accordingly, we see in Fig. 9 that the intensity of the radiated electric field is far more concentrated along the

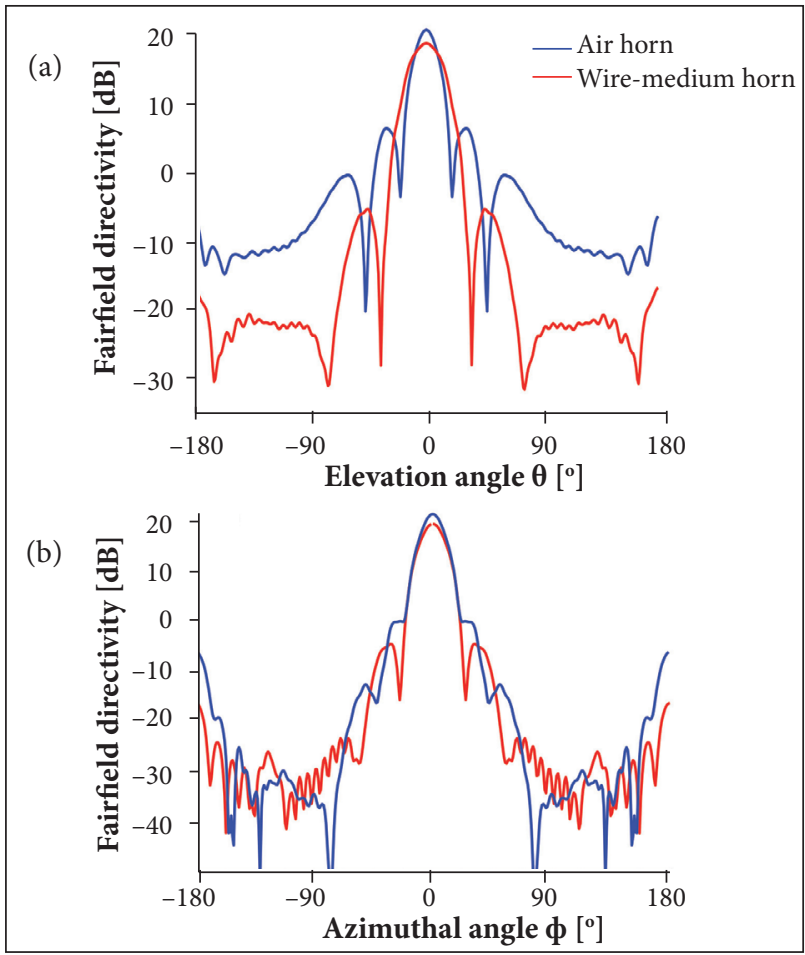

Figure 8. Far field radiation patterns in the (a) $E$ and (b) $H$ planes at $8.87 \mathrm{GHz}$. The 3-dB beamwidths, respectively, for the air horn and wire-medium antennas, are in (a) $16.0^{\circ}$ and $25.5^{\circ}$, and in (b) $16.2^{\circ}$ and $17.1^{\circ}$. axis of the wire-medium antenna. Note that, in contrast with Fig. 9b, the fields are more concentrated in the aperture of the loaded antenna in Fig. 9a. Similar behavior of refracted rays is shown in Fig. 5, which explains the reduction in the side lobe level in the far field radiation patterns.

The maximum directivity that can be obtained from an aperture of area $A$ is calculated as $D_{\text {max }}=4 \pi A / \lambda^{2}$, where $\lambda$ is the wavelength corresponding to the operation frequency. Since $A=113.74 \mathrm{~cm}^{2}$ from Fig. 6 and $\lambda=3.38 \mathrm{~cm}(8.87 \mathrm{GHz})$, $D_{\max }=21.0 \mathrm{~dB}$, which agrees with the simulated directivity $D=21.0 \mathrm{~dB}$ for the air horn antenna, with a simulated gain $G=20.8 \mathrm{dBi}$. For the loaded antenna, the simulated gain and directivity are $G_{\mathrm{L}}=18.6 \mathrm{dBi}$ and $\mathrm{D}_{\mathrm{L}}=18.9 \mathrm{~dB}$, respectively, which translates into a radiation efficiency of $\eta=G_{\mathrm{L}} / D_{\mathrm{L}}=98.4 \%$. The directivity is well estimated by the expression (Pozar 2005) $\mathrm{D}=32,400 / \Delta \phi \Delta \theta$, where $\Delta \phi$ and $\Delta \theta$ are the $3-\mathrm{dB}$ beamwidths in the two orthogonal planes of the main beam, in degrees. From Fig. 8 the angular widths are determined as $17.1^{\circ}$ and $25.5^{\circ}$, respectively, which gives $D=18.7 \mathrm{~dB}$ in close agreement with the simulated value.

At $11.86 \mathrm{GHz}$ (dip B in Fig. 7), the corresponding far-field radiation patterns are presented in Fig. 10. It is seen that the sidelobe level in the E-plane (Fig. 10a) is significantly reduced in comparison to that of the air horn antenna. The main lobe magnitudes for the air horn and wire-medium antennas are 24.0 and $22.0 \mathrm{~dB}$, whereas the $3-\mathrm{dB}$ beamwidths are, respectively, $10.8^{\circ}$ and $20.0^{\circ}$, so that the beamwidth of the wire-medium antenna is broadened. In the $H$-plane (Fig. 10b), the first and second lobes of the wire-medium antenna are increased relatively to those for the air horn. On the other hand, although the main

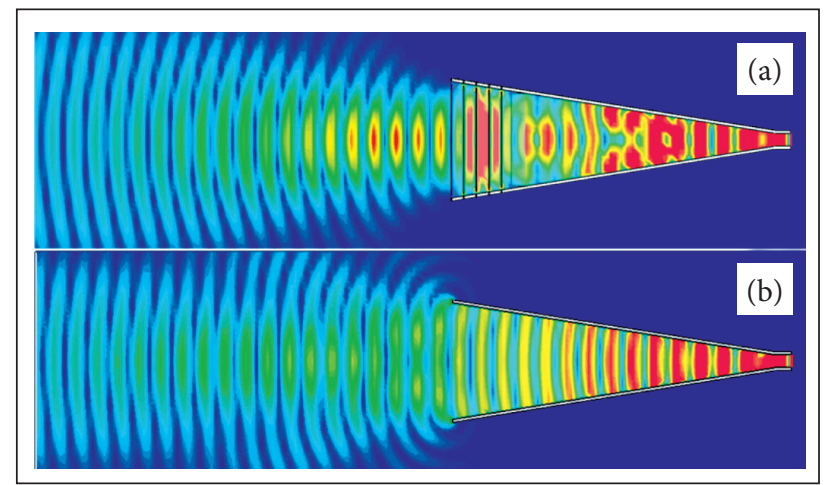

Figure 9. Electric field intensities radiated by (a) The wiremedium antenna and (b) The standard antenna operating at 8.87 GHz. The color scale ranges from $400 \mathrm{~V} / \mathrm{m}$ (red) to 0 (dark blue), where yellow means $300 \mathrm{~V} / \mathrm{m}$; green, $200 \mathrm{~V} / \mathrm{m}$; and light blue, 100V/m. 
lobe magnitude for the wire-medium antenna is decreased by $2.0 \mathrm{~dB}$ in comparison to the air horn, the $3-\mathrm{dB}$ beamwidth for the wire-medium antenna, namely $9.2^{\circ}$, is slightly narrower than that for the air horn, i.e. $10.2^{\circ}$.

Comparing the air horn radiation patterns, the $E$-plane sidelobe level (Fig. 10a) is significantly high in comparison with that in the $H$-plane (Fig. 10b). Nevertheless, as demonstrated in Fig. 10a, the wire medium was able to substantially reduce the high $E$-plane sidelobe level of the air horn, which constitutes one of the intrinsic drawbacks of such antennas.

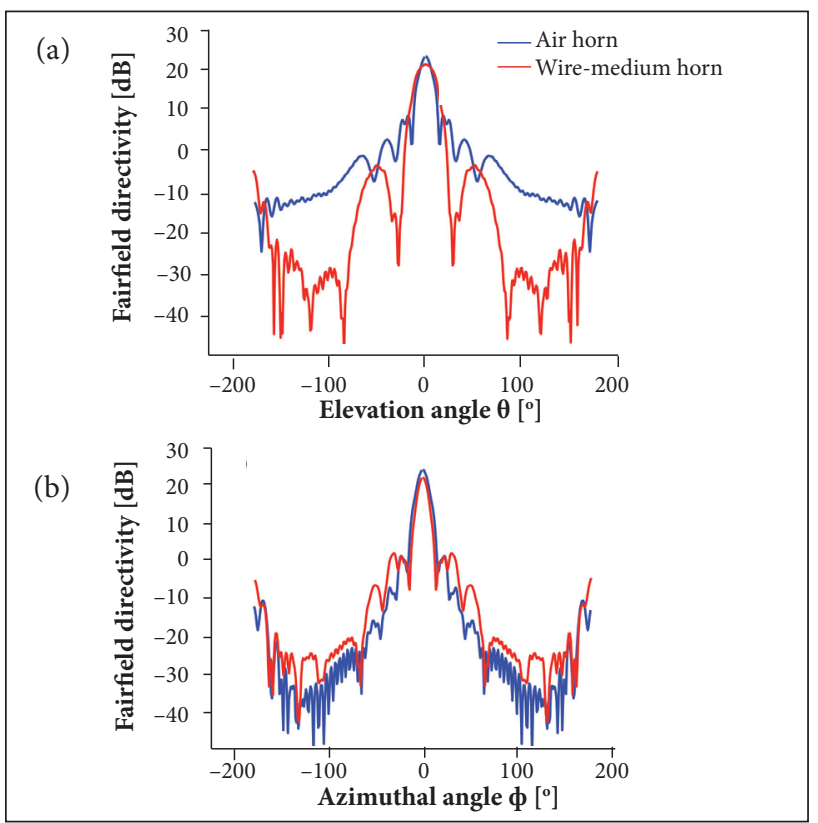

Figure 10. Far field radiation patterns in the (a) $E$ and (b) $H$ planes at $11.86 \mathrm{GHz}$. The 3-dB beamwidths, respectively, for the air horn and wire-medium antennas, are in (a) $10.8^{\circ}$ and $20.05^{\circ}$, and in (b) $10.2^{\circ}$ and $9.2^{\circ}$.

\section{CONCLUSIONS}

This report has demonstrated how a properly designed wire medium can reduce the side lobes of a commercial, standard X-band horn antenna. Supported by full-wave electromagnetic simulations, we have discussed analytical aspects and design considerations of a wire medium to enhance the radiation properties of a commercial X-band horn antenna, which is already optimized so that the effective aperture is quite close to the physical one. A design example has been given showing a noticeable reduction in the $E$-plane side lobes at 8.87 and $11.86 \mathrm{GHz}$. Such an enhancement is due to the near-zero refractive index effect providing a stronger concentration of energy in the central region of the loaded antenna radiation pattern. In the $E$-plane at $8.87 \mathrm{GHz}$, the wire-medium antenna exhibits a side lobe level $10 \mathrm{~dB}$ below that of the conventional antenna. This reduction, however, is obtained at the expense of a slight decrease of $2.4 \mathrm{~dB}$ in gain and directivity. Nevertheless, a major advantage of the wire-medium approach is that a conventional horn antenna may be easily and inexpensively modified to achieve improvement in side lobe performance which is a key-feature in radar, direction-finding equipment, and in systems to reject interference sources.

\section{ACKNOWLEDGMENTS}

We thank the Scientific and Technological Research Council of Turkey (TUBITAK) for providing the CST Microwave Studio software under the project grant number 112R032.

\section{REFERENCES}

Balanis CA (2005) Antenna Theory: Analysis and Design. New York: John Wiley \& Sons.

Barker-Jarvis J, Janezic MD, Riddle B, Holloway CL, Paulter NG, Blendell JE (2001) Dielectric and conductor-loss characterization and measurements on electronic packaging materials. Gaithersburg: National Institute of Standards and Technology, Technical Report 1520.

Barroso JJ, Tomaz A, Hasar UC (2014) Refractive properties of wiregrid metamaterials. J Electromagnet Wave 28(3):389-398. doi: 10.1080/09205071.2013.872057

Belov PA, Marqués R, Maslovski SI, Nefedov IS, Silveirinha M, Simovski CR, Tretyakov SA (2003) Strong spatial dispersion in wire media in the very large wavelength limit. Phys Rev B 67:1-4. doi: 10.1103/ PhysRevB.67.113103

Belov PA, Tretyakov SA, Viitanen AJ (2002) Dispersion and reflection properties of artificial media formed by regular lattices of ideally conducting wires. J Electromagnet Wave 16(8):1153-1170. doi: 10.1163/156939302X00688

CST Microwave Studio. Version 2014. Darmstadt: CST GmbH.

Datasheet Advanced Technical Materials, Inc., 2012. Horn Antennas. Standard Gain Horn.

Eleftheriades GV, Engheta N (2011) Metamaterials: fundamentals 
and applications in the microwave and optical regimes. $P$ IEEE 99(10):1618-1621. doi: 10.1109/JPROC.2011.2161808

Enoch S, Tayeb G, Sabouroux P, Guérin N, Vincent P (2002) A metamaterial for directive emission. Phys Rev Lett 89(21):1-4.

Franson SJ, Ziolkowski RW (2009) Confirmation of zero-n behavior in a high gain grid structure at millimeter-wave frequencies. IEEE Antennas Wireless Propag Lett 8:387-390. doi: 10.1109/ LAWP.2008.2002807

Hasar UC, Barroso JJ (2011) Retrieval approach for determination of forward and backward wave impedances of bianisotropic metamaterials. Prog Electromagn Res 112:109-124. doi: 10.2528/ PIER10112303

Hrabar S, Bonefacic D, Muha D (2009) Numerical and experimental investigation of basic properties of wire medium-based shortened horn antennas. Microw Opt Techn Let 51(11):2748-2753. doi: 10.1002/ mop. 24736

Lier E, Werner DH, Scarborough CP, Wu Q, Bossard JA (2011) An octave bandwidth negligible loss radiofrequency metamaterial. Nature Mater 10:216-222. doi: 10.1038/nmat2950

Lovat G, Burghignoli P, Capolino F, Jackson DR, Wilton DR (2006) Analysis of directive radiation from a line source in a metamaterial slab with low permittivity. IEEE Trans Antennas Propag 54(3): 1017-1030. doi: 10.1109/TAP.2006.869925

Maslovski SI, Tretyakov SA, Belov PA (2002) Wire media with negative effective permittivity: a quasi-static model. Microw Opt Techn Let 35(1):47-51. doi: 10.1002/mop.10512
Pozar DM (2005) Microwave engineering. Hoboken: John Wiley \& Sons.

Sahu B, Tripathi P, Singh R, Singh SP (2014) Dual segment rectangular dielectric resonator antenna with metamaterial for improvement of bandwidth and gain. Int J RF Microw C E 24(6):646-655. doi: 10.1002/mmce.20808

Semplak RA (1983) Measurements of selective near-in sidelobe reduction of pyramidal, horn-reflector antenna. AT\&T Tech J 62(3):595-605.

Simovski CR, Belov PA, Atrashchenko AV, Kivshar YS (2012) Wire metamaterials: physics and applications. Adv Mater 24(31):42294248. doi: 10.1002/adma.201200931

Tomaz A, Barroso JJ, Hasar UC, Orlando AJF (2013) Directivity enhancement of an X-band horn antenna loaded by a wire medium. Proceedings of the Progress in Electromagnetic Research Symposium; Stockholm, Sweden.

Tomaz A, Barroso JJ (2013) Refractive properties of artificial dielectrics consisting of a periodic wire medium. Proceedings of the IEEE International Symposium on Antennas and Propagation and National Radio Science Meeting; Orlando, USA

Wu Q, Pan P, Meng FY, Li LW, Wu J (2007) A novel flat lens horn antenna designed based on zero refraction principle of metamaterials. Appl Phys A-Mater 87(2):151-156. doi: 10.1007/s00339-0063820-9

Xiao Z, Xu H (2009) Low refractive metamaterials for gain enhancement of horn antenna. J Infrared Milli Terahz Waves 30:225232. doi: 10.1007/s10762-008-9449-3 